\title{
TELEPRESENCE EMERGENCY TRIAGE SYSTEM FOR EXTREME ENVIRONMENTS AND DISASTER RESPONSE
}

\author{
Kishan Patel ${ }^{1}$, Sabah Mohammed ${ }^{1}$ and Jinan Fiaidhi ${ }^{1}$ \\ ${ }^{1}$ Department of Computer Science, Lakehead University, Canada \\ ${ }^{1}\{$ kpatel14, jfiaidhi, mohammed $\} @$ lakeheadu.ca
}

\begin{abstract}
In this summary paper, we introduced the abstract details of our telepresence triage system. The design details of this telepresence triage system utilizes two emerging technologies including the real-time telecommunications and telepresence protocol WebRTC and the medical sensors attached to a Raspberry Pi 4. The system come up with a new idea where these Telepresence services can be implemented in a much efficient, economical and less sophisticated manner so that these services can be provided widely. The testing of the system prototype was encouraging and we are continuing our efforts to add more sensors to the present system.
\end{abstract}

Keywords - Remote Patient Monitoring, Physician Telepresence, Extreme Environments, Emergency Triage

\section{INTRODUCTION}

Emergency departments around the world use different triage systems to response to the severity of incoming patients' conditions and assign treatment priorities as well as for extreme environment or for responding to natural disasters. WHO defines an event as a disaster when "normal conditions of existence are disrupted and the level of suffering exceeds the capacity of the hazard-affected community to respond to it." In any given year, more than 100,000 people are killed in natural disasters around the globe, and millions more are injured or disabled [1]. The catastrophic earthquake that struck Haiti in 2010 and the destructive tsunami in the Indian Ocean in 2004 are recent examples that show the difficulties in providing medical care in the wake of such devastation. While a disaster by definition overwhelms response capabilities, a mass casualty incident (MCI) occurs more commonly and is defined as a situation that places a significant demand on medical resources and personnel [2]. Local response capabilities are not overwhelmed, but there are still a large number of patients requiring triage. A commonly occurring example of an MCI in the United States is a multiple vehicle or bus collision. Regardless of whether a situation is classified as a medical disaster or MCI, it requires rapid and effective triage methods. In order to optimize overall patient outcomes in a catastrophic situation, there is a shift from doing what is best for the individual patient to doing the greatest good for the largest number of people [3, 4]. A system of triage must be utilized to determine who will receive treatment and who will not, and the ethical considerations of allocating limited medical resources warrant in-depth discussion. With limited healthcare resources, innovations to improve patient safety and efficiency are needed. The implementation of physician tele-presence (PTP) in the field of medicine might be an innovation helpful in providing care. Currently, there is limited literature on the use of

Received: July 5, 2019

Reviewed: October 15, 2019

Accepted: October 29, 2019

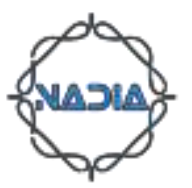


PTP in an ED setting or for the use at extreme or remote settings. To address this problem, this article describes a telepresence web-based application for monitoring patient vitals such as Heart rate, Oxygen level in blood, Temperature and blood pressure for remotely located patient using the emerging technology of the internet of things (IOT) and lightweight telepresence protocols like WebRTC ${ }^{1}$. The proposed triage system in this article requires a mobile device with internet connection with either chrome or Mozilla browser installed in it as well as sensors hooked to a Raspberry PI. The device is carried within the ambulance to conduct an initial assessment of the patient and the data can be shared with a health center in real-time fashion.

\section{THE TELEPRESENCE TRIAGE SYSTEM DESIGN:}

WebRTC is not just a video communications protocol, but rather a sophisticated telepresence protocol which can play a big role in public safety and designing a telepresence triage system. Figure 1 illustrated the capabilities of using WebRTC for PTP applications.

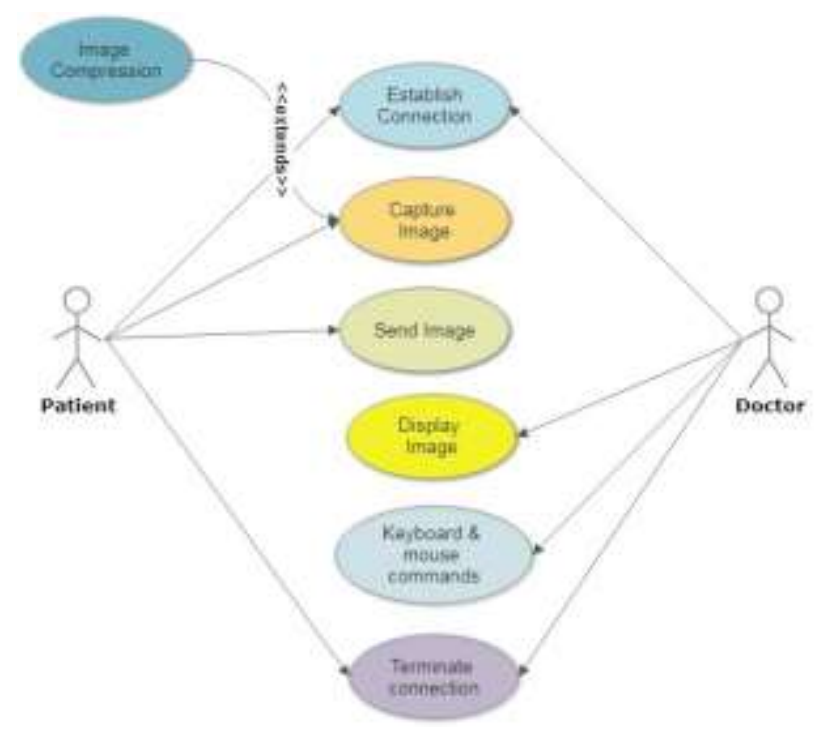

Fig. 1. General Telepresence Services for WebRTC

We assume that the paramedic had already an equipment that speaks WebRTC with a doctor or with an ED that implements our telepresence triage system. The paramedic starts a communication with the remote $\mathrm{ED} /$ doctor, and at the same time the wearable objects will start sending vital information in real-time to the ED, using WebRTC Data channel. The paramedic will at the same time apply first aides to the person in the simple case, and in case of serious injuries the $\mathrm{ED} /$ doctor can give real-time instructions, while transporting the patient. There are several usage scenarios of this communication setting. Figure 2 illustrates the first usage scenario in which the Emergency Doctor can view patient data remotely which includes body temperature, heartbeat, blood oxygen level and blood pressure. Here, first sensor data are sent to Raspberry PI which stores them into Database.

\footnotetext{
${ }^{1}$ https://webrtc.org/
} 


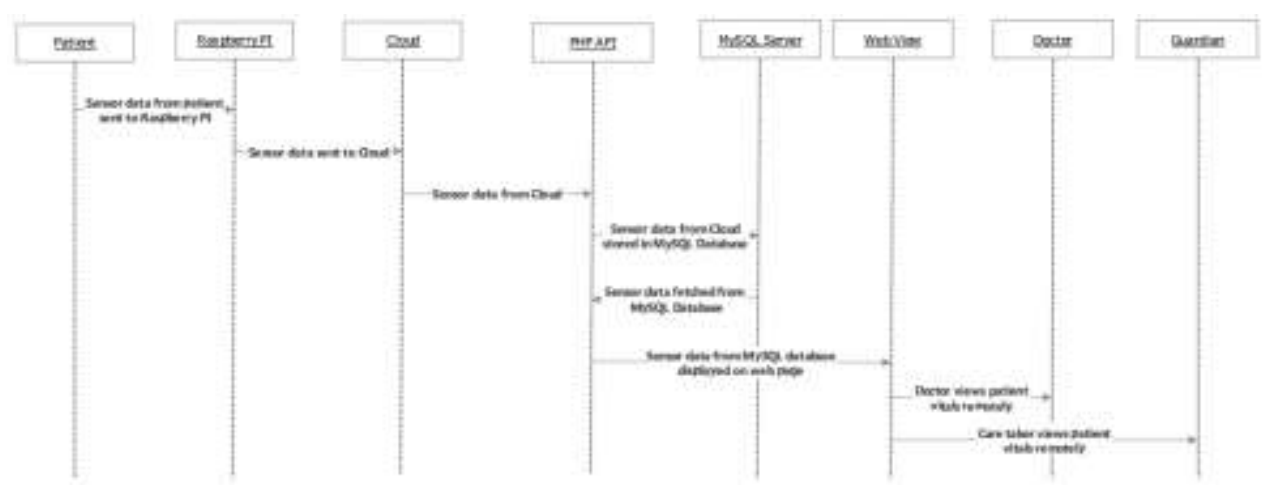

Fig. 2 ED Viewing Remote Patient Vitals using the Telepresence Triage.

Figure 3 illustrates another scenario in which the ED can use the telepresence triage and control the screen, keyboard and mouse of the remote patient's device.

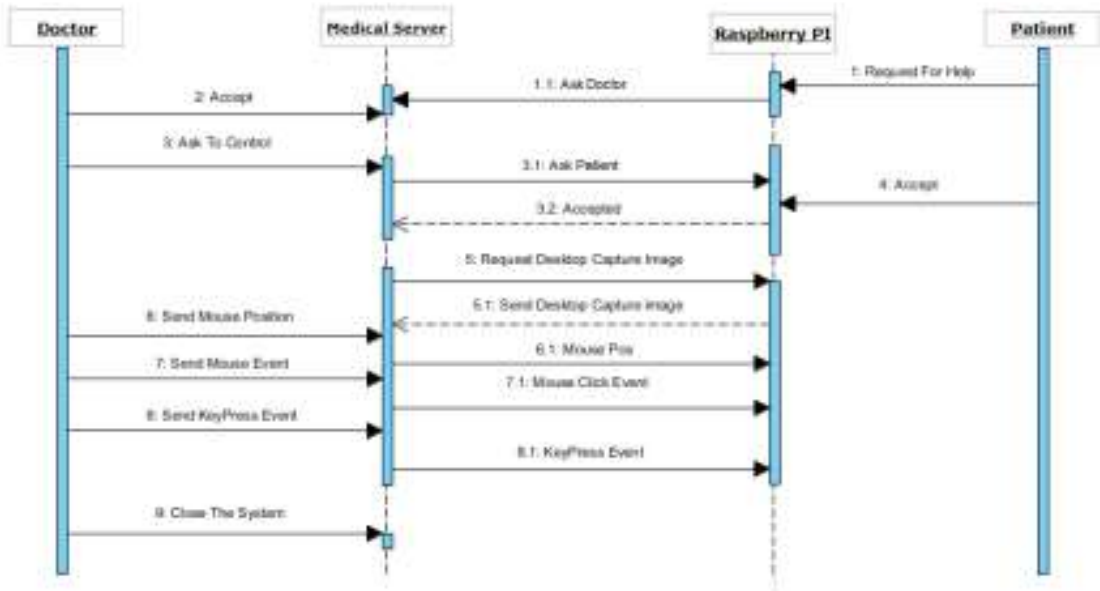

Fig. 3 ED Taking Control of the Telepresence Triage.

Figure 4 illustrate using the triage telepresence to communicate with the patient.

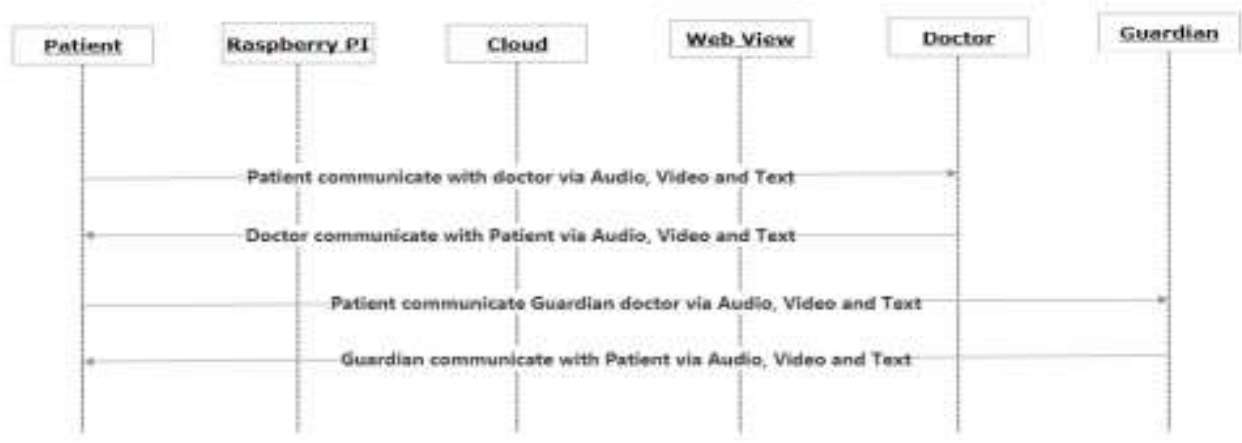

Fig. 4 ED Communicating with the Patient.

Figure 5 illustrate the telepresence triage sends a stress signal to ED when the patient vitals are below the safe thresholds. 


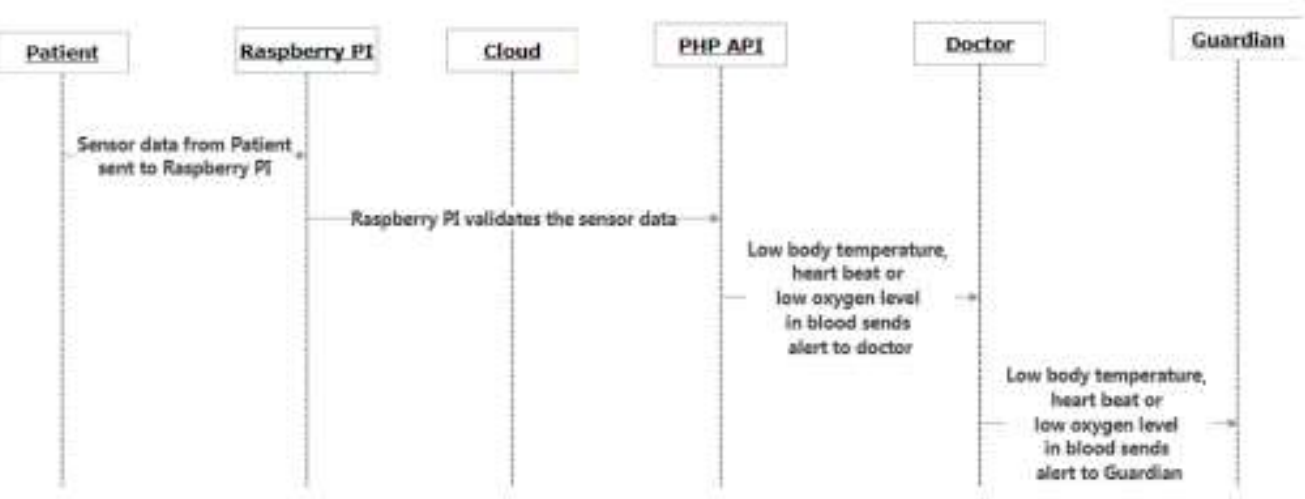

Fig. 5 The Telepresence Triage sends a Stress Signal when Vitals are Not Normal

Figure 6 illustrate the telepresence system sends an email to the ED reporting a device malfunction.

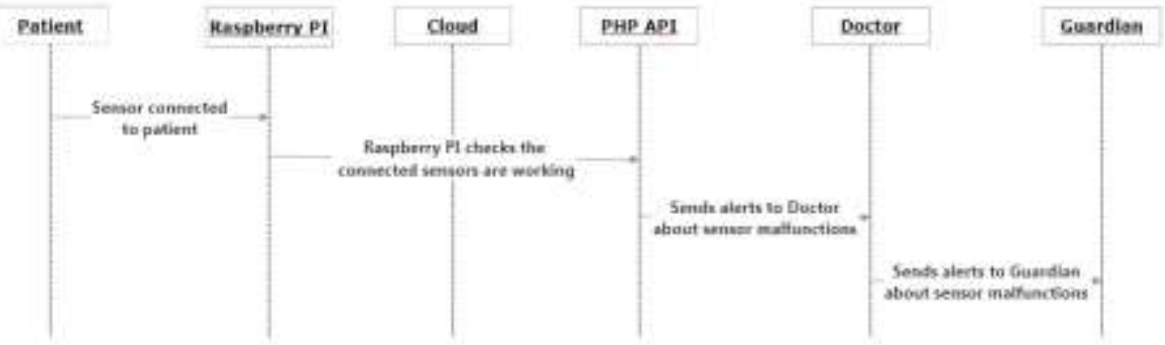

Fig. 6 The Telepresence Triage sends an Email Reporting a Mulfunction System.

Class diagram described in Figure 7 describes the structure of a system by showing the system's classes, their attributes, operations (or methods), and the relationships among objects.

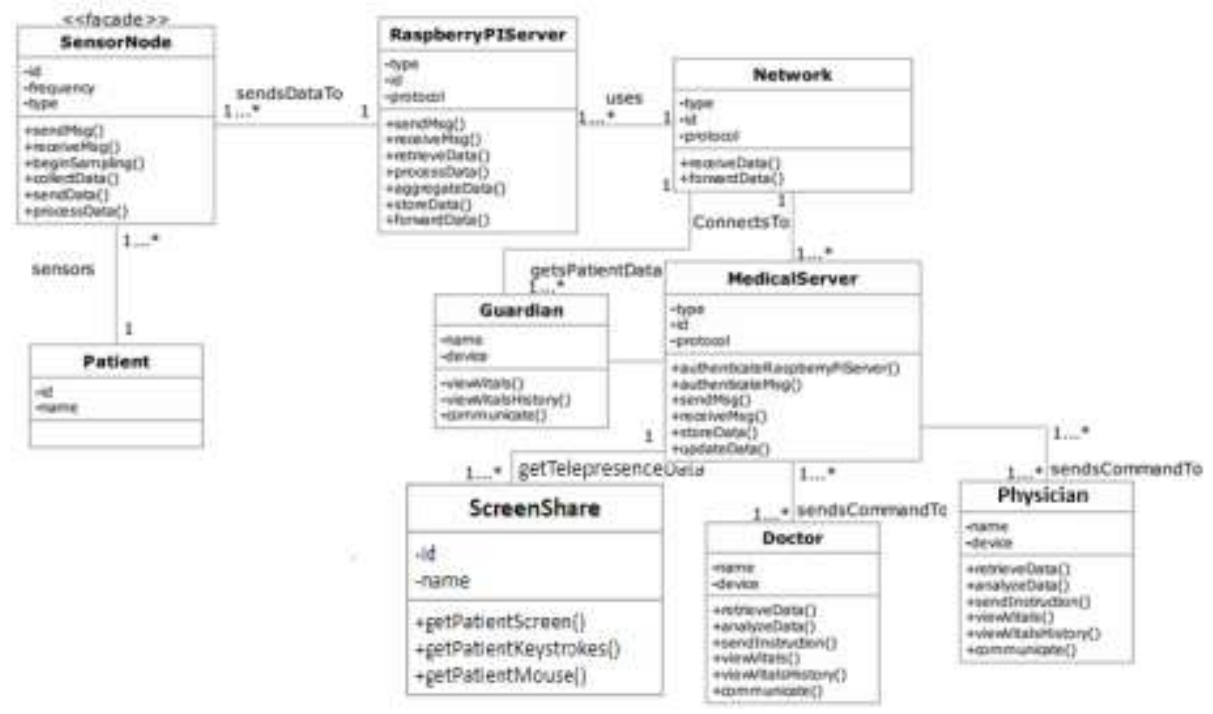

Fig. 7 The Telepresence Triage System Class Diagram. 


\section{WEBRTC TELEPRESENCE WEB PAGE:}

We have implemented a web platform using NodeJs. This platform can in one hand, establish a multimedia communication between two users just using their browsers. On the other hand, it enables users to access a set of predefined Things in his possession. Each user can then retrieve data from the different Things and send them to the remote user in real-time using WebRTC Data channel. The following are some screen shots of the Telepresence triage as viewed from the ED for a remote patient:

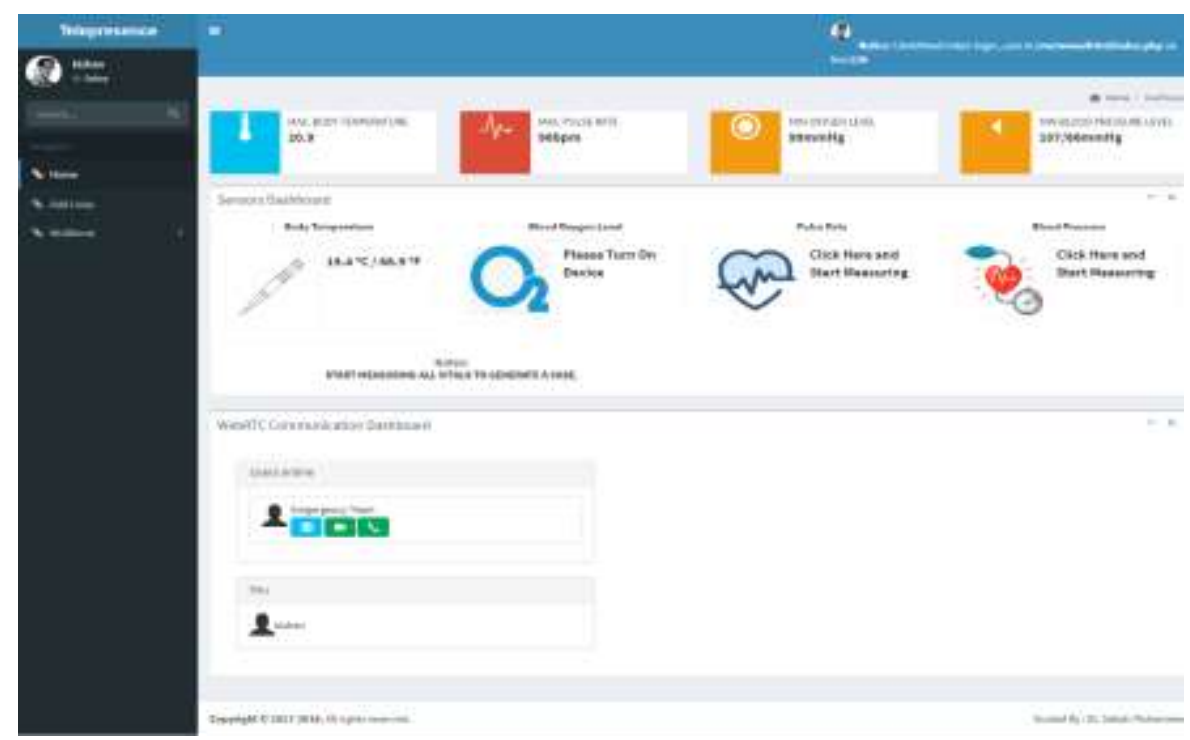

Fig. 8 The Telepresence Triage Web Page.

Python has been used for reading and processing data from the sensor and sends the vital information to php. The code is executed every second and if vital information is available from the patients. The Telepresence Triage algorithm calculates the abnormal vitals and opens a case which sends alerts to general practitioner or emergency team. The overall telepresence triage design is illustrated in Figure 9.

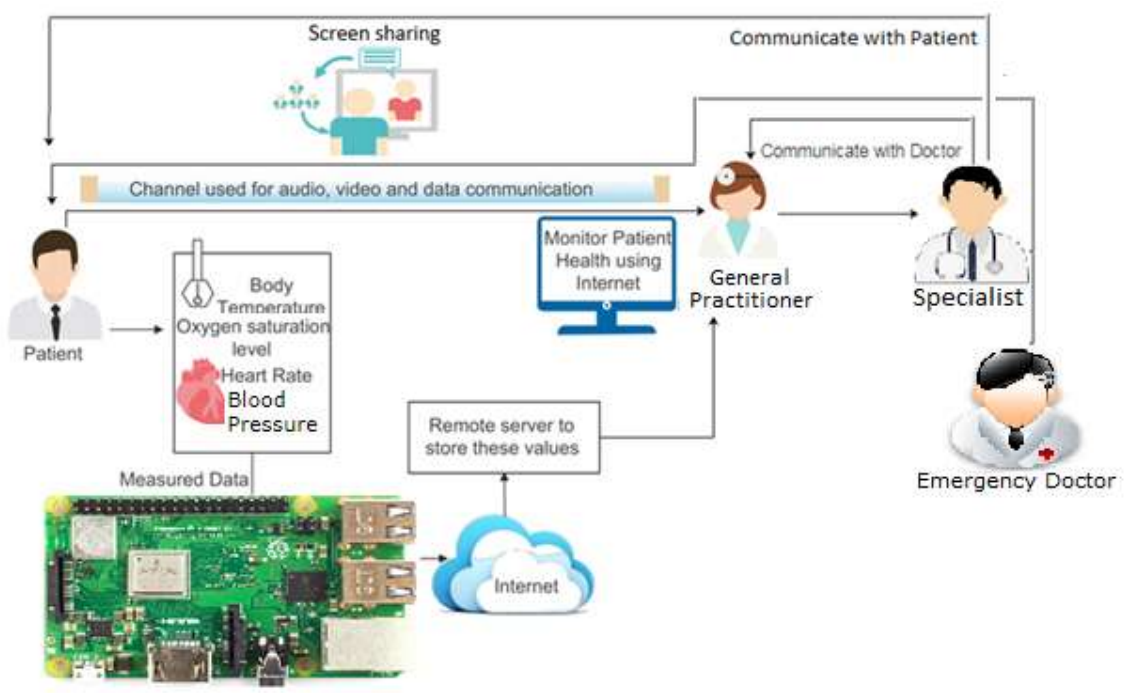

Fig. 9 The Overall Design of the Telepresence Triage System. 


\section{CONCLUSIONS}

This case study paper present an ongoing work for the creation of a telepresence triage Framework specially conceived for remote examination of patients. Though the different use cases, we illustrated the various means of achieving telepresence. WebRTC and Raspberry Pi 4 has been used with sensors like DS18B20 Temperature Sensor, Sunrom Blood Pressure Sensor and the CONTEC CMS50D Plus Fingertip Blood Oxygen Pulse Oximeter. Our initial tests shows the reliability and scalability of this system as well as the practical use for extreme environments. We are continuing our work to add more medical sensors to our system that can provide the ED with other medical data on the remote patients.

\section{ACKNOWLEDGMENTS}

This is a summary paper is part of the first author MSc Project research within the Smart Health FabLab at Lakehead University.

\section{REFERENCES}

[1] World Health Organization Regional Office for the Western Pacific. Factsheet: care of the dead in disasters. www.wpro.who.int/media_centre/fact_sheets/fs_20061207.htm. Accessed May 11, 2010.

[2] Noji EK. Disaster medical services. In: Tintinalli JE, Ruiz E, Krome RL, eds. Emergency Medicine: A Comprehensive Study Guide. 5th ed. New York: McGraw-Hill; 2000: 20-31.

[3] Koenig KL, Cone DC, Burstein JL, Camargo CA. Surging to the right standard of care. Acad Emerg Med. 2006;13(2):195-198.

[4] Frykberg ER. Medical management of disasters and mass casualties from terrorist bombings: how can we cope? J Trauma. 2002;53(2):201-212.

[5] Mehta D, Siddiqui MF, Javaid AY. Recognition of emotion intensities using machine learning algorithms: A comparative study. Sensors. 2019 Jan;19(8):1897.

[6] Chao H, Dong L, Liu Y, Lu B. Emotion Recognition from Multiband EEG Signals Using CapsNet. Sensors. 2019 Jan;19(9):2212.

[7] Zheng WL, Dong BN, Lu BL. Multimodal emotion recognition using EEG and eye tracking data. In2014 36th Annual International Conference of the IEEE Engineering in Medicine and Biology Society 2014 Aug 26 (pp. 5040-5043).

[8] Li Y, Huang J, Zhou H, Zhong N. Human emotion recognition with electroencephalographic multidimensional features by hybrid deep neural networks. Applied Sciences. 2017 Oct 13;7(10):1060.

[9] Lopes da Silva, Gonçalves, and de Munck (2009). "Electroencephalography (EEG). Encyclopedia of Neuroscience", 849-855.

[10] J. DellaBadia Jr., et al. "Assessment and cost comparison of sleep-deprived EEG, MRI, and PET in the prediction of surgical treatment for epilepsy, " Seizure, vol. 11, no. 5, pp. 303-309, 2002.

[11] K. Ishino and M. Hagiwara, "A Feeling Estimation System Using a Simple Electroencephalograph," Proc. ICSMC, vol.5, pp. 4204-4209, 2003.

[12] Y. Chavhan, M. Dhore, and P. Yesaware, "Speech Emotion Recognition using Support Vector Machine," International Journal of Computer Applications, vol. 1, no. 20, pp. 8-11, 2010. Available: 10.5120/431-636.

[13] V. H. Anh, M. N. Van, B. B. Ha, and T. H. Quyet, "A real-time model based Support Vector Machine for emotion recognition through EEG," 2012 International Conference on Control, Automation and Information Sciences (ICCAIS), 2012.

[14] R. Jenke, A. Peer, and M. Buss, "Feature Extraction and Selection for Emotion Recognition from EEG," IEEE Transactions on Affective Computing, vol. 5, no. 3, pp. 327-339, 2014.

[15] R. Nivedha, M. Brinda, D. Vasanth, M. Anvitha, and K. V. Suma, "EEG based emotion recognition using SVM and PSO," 2017 International Conference on Intelligent Computing, Instrumentation and Control Technologies (ICICICT), 2017.

[16] Koelstra S, Muhl C, Soleymani M, Lee JS, Yazdani A, Ebrahimi T, Pun T, Nijholt A, Patras I. Deap: A database for emotion analysis; using physiological signals. IEEE transactions on affective computing. 2011 Jun 9;3(1):18-31. 